\title{
A IMPORTÂNCIA DA MATEMÁTICA DISCRETA NA FORMAÇÃO DE PROFESSORES DE MATEMÁTICA
}

\author{
THE IMPORTANCE OF DISCREET MATHEMATICS \\ IN THE TRAINING OF MATHEMATICS TEACHERS \\ Rubens Vilhena Fonseca ${ }^{1}$ \\ Teodora Pinheiro Figueroa ${ }^{2}$ \\ Andreza Thalia Menezes Monteiro ${ }^{3}$
}

\section{Resumo}

Esta pesquisa, de cunho teórico-qualitativo, tem como foco principal mostrar a importância da Matemática Discreta na formação de professores de matemática. Para isso realizamos uma pesquisa teórica em trabalhos científicos e, apresentamos algumas discussões sobre questões matemáticas relacionadas com a Aritmética dos Inteiros, as quais comprovam a importância do raciocínio discreto. Nossa investigação tem como foco a seguinte questão de pesquisa: Quais as contribuições da Matemática Discreta na formação de professores? É necessário repensar sobre praxeologias que poderiam favorecer a um tipo de raciocínio por meio do qual os estudantes discorreriam sobre uma maneira de pensar, na perspectiva da Matemática Discreta?A partir de resultados de pesquisas apresentamos um contexto histórico das Equações Diofantinas Lineares e do Algoritmo de Euclides, Conexões Matemáticas: Ensino Superior x Escola Básica e, Teorias Matemáticas relativas a estes saberes, de modo a mostrar que existe a possibilidade de discussões sob o ponto de vista da Matemática Discreta entre o ensino superior e a escola básica na formação de professores. Os trabalhos de pesquisas investigados validaram a nossa proposta.

Palavras-chave: Equações Diofantinas. Educação Básica. Algoritmo de Eucldes. Matemática Discreta

\footnotetext{
${ }^{1}$ Universidade do Estado do Para (UEPA) - e-mail: rubens.vilhena @ uepa.br

${ }^{2}$ Universidade Tecnológica Federal do Paraná (UTFPR-PB) - e-mail: teodora.pinheiro@gmail.com

${ }^{3}$ Universidade do Estado do Para (UEPA) - e-mail: andrezathaliamn@ gmail.com
} 
A importância da Matemática Discreta na formação de professores de Matemática

\begin{abstract}
This theoretical and qualitative research, has as main focus to show the importance of Discrete Mathematics in the formation of mathematics teachers. For this we carry out a theoretical research in scientific works and, we present some discussions on mathematical questions related to the arithmetic of integers, which prove the importance of discreet reasoning. Our investigation focuses on the following research question: What are the contributions of Discrete Mathematics in teacher education? Is it necessary to rethink about praxeologies that could favor a type of reasoning through which students would discuss a way of thinking, in the perspective of Discrete Mathematics? From research results we present a historical context of Linear Diophantine Equations and Euclid's Algorithm, Mathematical Connections: Higher Education x Basic School and, Mathematical Theories related to this knowledge, in order to show that there is the possibility of discussions under the point from the point of view of Discrete Mathematics between higher education and basic school in teacher training. The research work investigated validated our proposal.
\end{abstract}

Key words: Diophantine Equations. Basic Education. Euclid's Algorithm. Discrete Mathematics.

\title{
Introdução
}

Em matemática há duas grandes correntes. A Matemática Discreta que trata de assuntos como indução, recursão, combinatória, e em geral tudo o que se refere à aritmética dos números inteiros. E a Matemática Contínua, a que lida com a ideia de função, com a geometria, com derivadas e integrais e, em geral, a tudo que se refere a álgebra dos números reais.

Utilizamos os termos discreto e continuo em referência, respectivamente, a duas das ações básicas da Matemática: contar e medir. Existem, como sabemos, certas grandezas chamadas contáveis, que são objetos de contagem, como o número de livros em uma prateleira. Outro tipo de grandeza é formado por aquelas quantidades que são passíveis de medida, como a largura de uma folha de papel A4, ou o peso de uma caneta. O primeiro tipo de grandeza é chamado discreto. Grandezas discretas são as que se prestam a contagem. Já o segundo tipo é chamado contínuo, e se refere às medidas. (BROLEZZI, 1996).

A ideia principal é que a Matemática Discreta é o estudo de estruturas matemáticas que são "discretas" em contraste com as 
A importância da Matemática Discreta na formação de professores de Matemática

"contínuas". Estruturas discretas são configurações, isso pode ser caracterizado com um conjunto finito ou contável de relações. (OUVRIER-BUFFET, 2014, p. 181).

Os grandes avanços tecnológicos digitais que ocorreram nas décadas finais do século $\mathrm{XX}$ e, que continuam a nos causar admiração e provocam grandes mudanças sociais, colocaram a Matemática Discreta como a "matemática do nosso tempo". (DOSSEY, 1991).

Além disso, foi incentivada nas escolas primárias e secundárias nos Estados Unidos, uma introdução à Matemática Discreta através das recomendações dos documentos americanos National Council of Teachers of Mathematics (NCTM) no Curriculum and Evaluation Standards for School Mathematics (NCTM, 1989). Estes documentos incluíram a Matemática Discreta como um conteúdo padrão para o nível médio (MENINO, 2013). Dossey (1991) forneceu o cenário histórico para estas recomendações. Ele descreve o surgimento da Matemática Discreta como uma área separada de estudo a partir do final dos anos 1960 com textos influentes que aparecem no nível superior de graduação ao longo da década de 1970 .

Uma publicação recente que analisa o futuro da matemática, As Ciências Matemáticas em 2025 (Comitê de Ciências Matemáticas em 2025, 2013, p.77) identifica dois novos direcionadores da matemática: computação e big data. Estes direcionadores descrevem como a Matemática Discreta desempenha um papel importante em algoritmos matemáticos para processamento de dados, em sistemas dinâmicos em ecologia, em redes industriais e de humanidades e em otimização discreta.

Considerando que a Matemática Discreta é a matemática para o nosso tempo, uma vez que muitos dos nossos alunos são dessa geração da era digital, o objetivo deste trabalho é discutir alguns aspectos da aritmética dos inteiros que podem ser explorados na educação básica (ensino fundamental e médio). 
A importância da Matemática Discreta na formação de professores de Matemática

Conforme a Base Nacional Comum Curricular (BNCC):

Da mesma forma que na fase anterior, a aprendizagem em Matemática no Ensino Fundamental - Anos Finais também está intrinsecamente relacionada à apreensão de significados dos objetos matemáticos. Esses significados resultam das conexões que os alunos estabelecem entre os objetos e seu cotidiano, entre eles e os diferentes temas matemáticos e, por fim, entre eles e os demais componentes curriculares. Nessa fase, precisa ser destacada a importância da comunicação em linguagem matemática com o uso da linguagem simbólica, da representação e da argumentação. (BRASIL, 2017, p.298).

Do ponto de vista de que é de fundamental importância para o ensino e a aprendizagem de matemática a apreensão dos significados dos objetos matemáticos e, de que para isso se faz necessário que conexões sejam estabelecidas entre os mesmos e, principalmente entre os demais componentes curriculares, nossa pesquisa apresenta um contexto histórico das Equações Diofantinas Lineares e do Algoritmo de Euclides, Conexões Matemáticas: Ensino Superior x Escola Básica e, Teorias Matemáticas relativas a estes saberes, de modo a mostrar que existe a possibilidade de discussões sob o ponto de vista da Matemática Discreta entre o ensino superior e a escola básica na formação de professores. Acredita-se que este tipo de abordagem contribuirá na atuação docente do futuro professor e, as interações destes com os alunos em seu processo de transposição didática.

A seguir apresentaremos uma visão da Matemática Discreta na perspectiva de suas contribuições no ensino e aprendizagem de matemática 
A importância da Matemática Discreta na formação de professores de Matemática

\section{Matemática Discreta - Contribuições para o ensino e aprendiza- gem de matemática}

A Matemática Discreta apresenta conteúdos importantes para aplicações da matemática escolar - combinatória e raciocínio combinatório, recursão e pensamento recursivo, tomadas de decisões corretas, lógica e prova, entre outros.

Os conteúdos da Matemática Discreta estão presentes nos currículos, mas de forma implícita no sentido de apresentação de conexões existentes entre eles. Se estas conexões não são explícitas e, se os alunos não se apropriam delas, acredita-se que a falta das mesmas pode ser considerada como obstáculos epistemológicos para o ensino e aprendizagem desses conteúdos e, mais do que isso, a falta de apropriação e significação dos mesmos não levarão os alunos a reconhecer a aplicabilidade da Matemática Discreta em situações de seu cotidiano, por exemplo.

Muito se fala sobre "aplicação da matemática", mas pouco se discute sobre as conexões necessárias para que isto ocorra.

Mas, com relação a inserção da Matemática Discreta, nos ensinos fundamental e médio, existe uma discussão muito presente em entidades norte-americanas, como por exemplo, no Conselho Nacional dos Professores de Matemática (NCTM, 2000, p.31), o qual reconhece a Matemática Discreta como uma ferramenta interessante e, que poderia estar em evidência em diversos tópicos do ensino fundamental e médio e, consequentemente fazer parte do currículo escolar.

Segundo Debellis e Rosenstein (2004), conforme observado na Declaração de Visão da conferência DIMACS (Discrete Mathematics and Theoretical Computer Science) de 1992:

Nos últimos 30 anos, a Matemática Discreta cresceu rapidamente e tornou-se uma área significativa da matemática. Cada vez mais, Matemática Discreta é a matemática que é usada pelos 
A importância da Matemática Discreta na formação de professores de Matemática

tomadores de decisão em negócios e governo; por trabalhadores de áreas como telecomunicações e computação que dependem da transmissão de informações; e por aqueles em muitas profissões em rápida mudança envolvendo cuidados de saúde, biologia, química, fabricação automatizada, transporte, etc. Cada vez mais, a Matemática Discreta é a linguagem de um grande corpo da ciência e está por trás das decisões que os indivíduos terão que tomar em suas próprias vidas, em suas profissões e como cidadãos." (DEBELLIS; ROSENSTEIN, 2004, p.48).

De acordo com Debellis e Rosenstein (2004), os alunos devem ser capazes de resolver problemas de contagem - como encontrar o número de pizzas diferentes que usam quatro das oito coberturas, e aplicar estratégias de contagem para resolver problemas de probabilidade. Eles devem ser capazes de modelar e resolver problemas de crescimento, como o número de animais em um habitat, utilizando procedimentos iterativos. Além disso, os autores comentam que da perspectiva deles a Matemática Discreta não é apenas um conteúdo de matemática com o qual os alunos devem se tornar familiar. Para escolas de ensino fundamental e médio, a Matemática Discreta deve ser mais do que apenas uma coleção de novos tópicos matemáticos interessantes.

Debellis e Rosenstein (2004) comentam, em sua pesquisa, sobre os resultados do trabalho de Rosenstein (1997), ou seja, de uma experiência em relação ao LP-DM (Programa de Liderança em Matemática Discreta) da Universidade de Rutgers, o qual forneceu programas de desenvolvimento profissional para professores do ensino fundamental e médio.

Os participantes relataram mudanças nas salas de aula,em seus alunos e em si mesmos. Seus sucessos nos ensinou que a Matemática Discreta não era apenas mais uma parte do currículo. Muitos participantes relataram sucesso com uma variedade de estudantes em uma variedade de níveis, demonstraram um novo entusiasmo por ensinar de novas maneiras e proselitizaram este entusiasmo entre seus colegas e administradores".(ROSENSTEIN, 1997, apud DEBELLIS e ROSENSTEIN, 2004, tradução nossa). 
A importância da Matemática Discreta na formação de professores de Matemática

Aqui no Brasil, na BNCC (BRASIL, 2017, p.540) na seção Matemática e suas tecnologias no ensino médio: competências específicas e habilidades, no quadro referente às Habilidades da Competência 5:

Competência 5: Investigar e estabelecer conjecturas a respeito de diferentes conceitos e propriedades matemáticas, empregando estratégias e recursos, como observação de padrões, experimentações e diferentes tecnologias, identificando a necessidade, ou não, de uma demonstração cada vez mais formal na validação das referidas conjecturas. (BRASIL, 2017, p. 540).

existem comentários sobre domínios discretos na organização curricular Números e Probabilidade, como por exemplo, Figura 1:

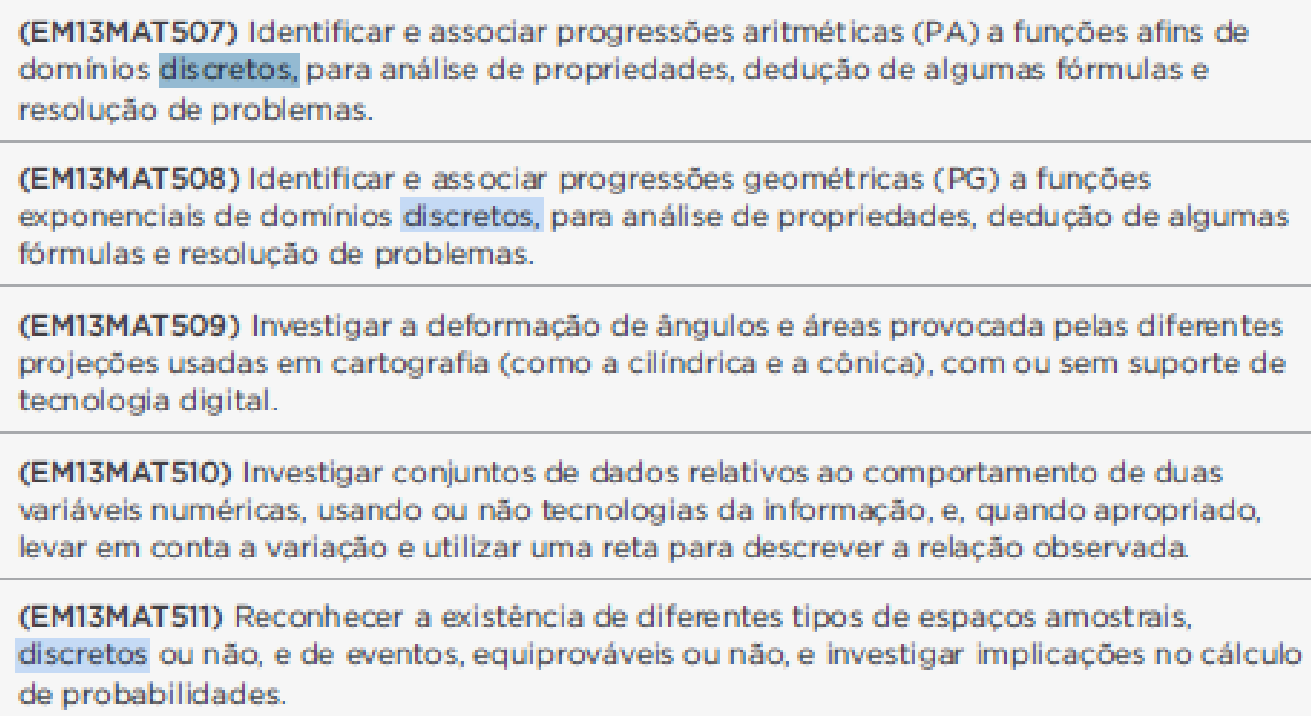

(EM13MAT509) Investigar a deformação de ângulos e áreas provocada pelas diferentes projeçōes usadas em cartografia (como a cilíndrica e a cônica), com ou sem suporte de tecnologia digital.

(EM13MAT510) Investigar conjuntos de dados relativos ao comportamento de duas variáveis numéricas, usando ou não tecnologias da informação, e, quando apropriado, levar em conta a variação e utilizar uma reta para descrever a relação observada.

(EM13MAT511) Reconhecer a existência de diferentes tipos de espaços amostrais, discretos ou não, e de eventos, equiprováveis ou não, e investigar implicações no cálculo de probabilidades.

Figura 1. Habilidades

Fonte: BNCC (BRASIL, 2017, p.540)

Mas, não é explícito como implementar a Matemática Discreta nestes assuntos. E, dessa forma, fica evidente o papel da universidade na formação dos professores de Matemática. 
Diante deste fato, surge a seguinte questão: Será que os cursos de Licenciatura em Matemática estão discutindo sob esta perspectiva, quais as contribuições destas discussões?

Nossa visão da Matemática Discreta, como mostrada a partir de pesquisas realizadas, é que a mesma é um veículo para dar aos professores uma nova forma de pensar sobre temas matemáticos e uma nova estratégia para engajar os alunos no estudo da matemática.

A seguir, apresentaremos uma discussão sobre o contínuo e o discreto com ênfase na matemática escolar.

\section{O discreto e o contínuo - uma discussão necessária no ensino}

O conjunto discreto usual na escola básica é o conjunto dos números inteiros $Z$, assim como, o conjunto contínuo padrão é o conjunto dos números reais $R$.

As características intrínsecas destes conjuntos determinam aspectos importantes na discussão sobre as diferenças entre uma abordagem discreta e contínua na resolução de determinados tipos de problemas matemáticos.

A seguir apresentaremos dois tipos de problemas e uma análise destes aspectos inerentes a estes dois tipos de abordagens.

Problema 1: Determine o valor de $\mathrm{x}$ na equação $3 \mathrm{x}-5=2$.

Tipo de Resolução 1: Resolução a partir de manipulações algébricas usando os princípios aditivos e multiplicativos, os quais reduzem uma equação em outra equivalente, obtendo-se assim $x=7 / 3$

A resolução 1 tem como foco um algoritmo de resolução, mas este tipo de resolução não deixa explícito o raciocínio do aluno e, se de fato ele se apropriou dos conceitos implícitos nas manipulações algébricas. 
Este tipo de resolução nos leva a seguinte questão: Será que os alunos seriam capazes de resolver este problema de outra maneira?

Tipo de Resolução 2: Resolução a partir de um raciocínio discreto para verificar se há um inteiro x que satisfaça a equação, como mostrado na Tabela 1.

Tabela 1. Dados da resolução

\begin{tabular}{|c|c|c|c|c|c|c|c|}
\hline $\boldsymbol{x}$ & -3 & -2 & -1 & 0 & 1 & 2 & 3 \\
\hline $\mathbf{3 x}-\mathbf{5}$ & -14 & -11 & -8 & -5 & -2 & 1 & 4 \\
\hline
\end{tabular}

Fonte: Autores

Em um primeiro momento poderemos considerar alguns valores inteiros de $\mathrm{x}=1,2$ e 3 e obter os resultados $-2,1$ e 4conforme a Tabela 1, para concluir que não é possível obter o 2 , se forem considerados apenas valores discretos.

No tipo de resolução 2, o raciocínio do aluno fica mais evidente. Porém duas coisas poderiam acontecer: a) atribuir apenas valores de " $x$ " inteiros na tabela, e/ou b) perceberem a partir da tabela que o valor de $\mathrm{x}$ para o qual $3 \mathrm{x}-5=2$ está entre os valore de $\mathrm{x}=2$ e $\mathrm{x}=3$ e, assim começar a atribuir valores do conjunto contínuo dos racionais positivos até encontrar o valor de $\mathrm{x}=7 / 3$.

A resolução 2 é característico de um tipo de raciocínio discreto, o qual não é contemplado na Unidade Temática Álgebra do $7^{\circ}$ ano da Base Nacional Comum Curricular (BNCC) (BRASIL, 2017, p. 543). 
A importância da Matemática Discreta na formação de professores de Matemática

Quadro 1: Unidade Temática álgebra

\begin{tabular}{|c|l|}
\hline \multicolumn{1}{|c|}{ Objeto de Conhecimento } & \multicolumn{1}{|c|}{ Habilidades } \\
\hline Equações Polinomiais do $1^{\circ}$.grau & $\begin{array}{l}\text { (EF07MA18) Resolver e elaborar pro- } \\
\text { blemas que possam ser representados } \\
\text { por equações polinomiais de } 1^{\circ} . \text { Grau, } \\
\text { redutiveis à forma ax }+ \text { b }=\text { c, fazendo } \\
\text { uso das propriedades de igualdade. }\end{array}$ \\
\hline
\end{tabular}

Fonte: Autores

Dessa forma, podemos dizer que o raciocínio discreto não é contemplado na BNCC para a resolução do tipo de Problema 1 na educação básica, por exemplo. E, este fato pode ser um obstáculo epistemológico em problemas de contagem.

Problema 2: Esboçar o gráfico da função $y=2 x-6$

Tipo de Resolução 1: Atribuir dois valores discretos, por exemplo, para $\mathrm{x}$ e, assim obter os respectivos valores discretos para y e, traçar o gráfico contínuo conforme a Figura 2.

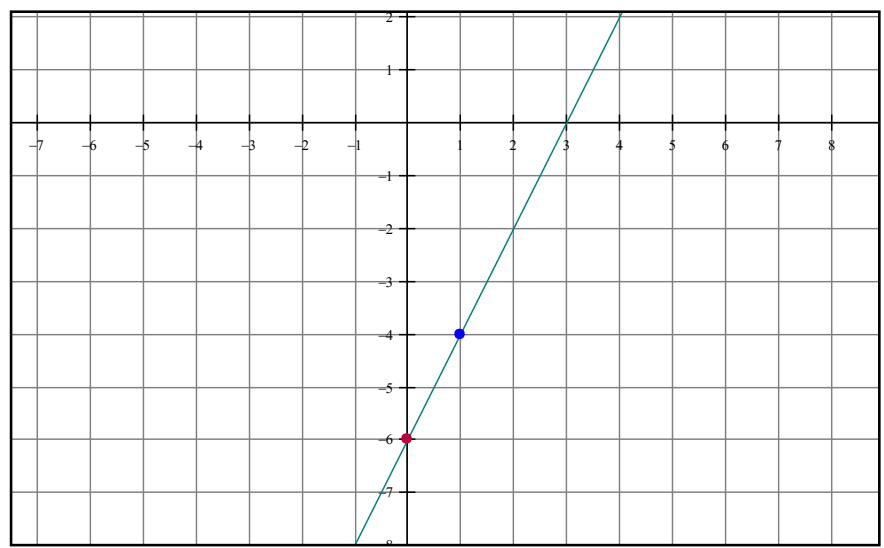

Figura 2: Reta $\mathrm{y}=2 \mathrm{x}-6$

Fonte: Autores

Matemática \& Ciência, v. 3, n. 1, p. 8-37, jun. 2020 - ISSN 2674-9416 
A resolução 1, atribuindo apenas dois valores para $\mathrm{x}$, e obtendo os respectivos valores para y, deixa implícito, por exemplo, a ideia de que uma reta tem infinitos números e, por isso, a característica de continuidade da reta. Diante deste fato, surge a seguinte questão: Será que os alunos ao resolverem dessa forma, compreendem a ideia de variável contínua ou discreta? Isso é algo que este tipo de resolução não nos permite saber.

Tipo de Resolução 2: Uso do raciocínio discreto atribuindo vários valores discretos para $\mathrm{x}$, determinando os respectivos valores de $\mathrm{y}$ e, assim traçar um gráfico com os respectivos pontos $(\mathrm{x}, \mathrm{y})$, Figura 3.

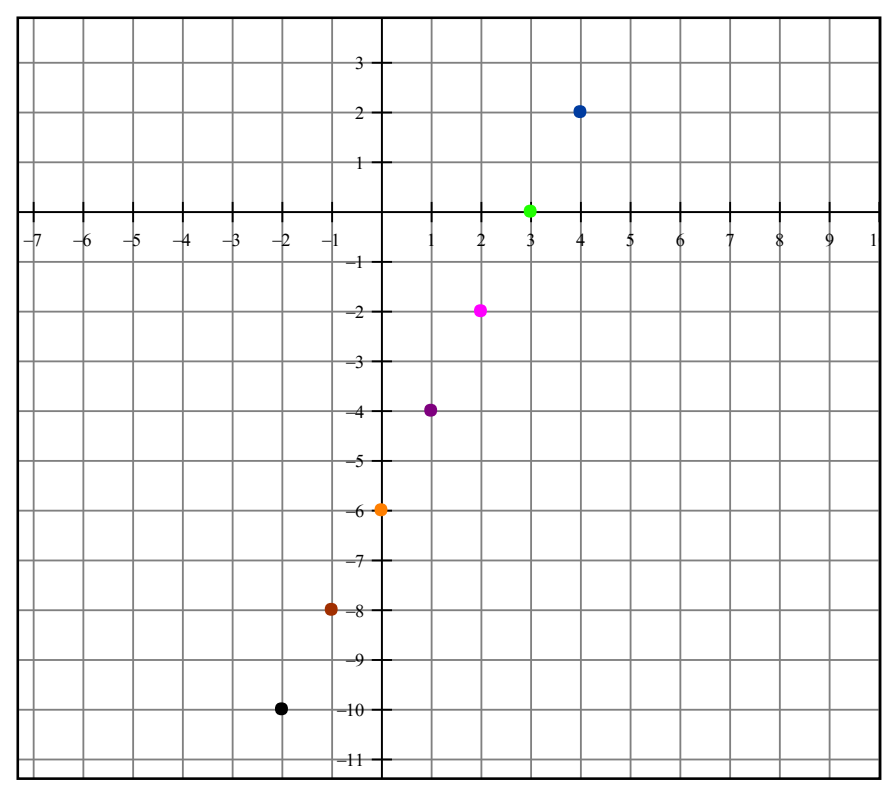

Figura 3: Gráfico de Pontos discretos da reta $y=2 x-6$ Fonte: Autores 
A importância da Matemática Discreta na formação de professores de Matemática

No tipo de resolução 2, fica explícito o raciocínio discreto e, as coordenadas cartesianas exibem os valores de um conjunto de dados característicos da reta dada.

Pode-se concluir a partir desses dois tipos de problemas que o tipo de resolução 1 em ambos os problemas explora mais aspectos algébricos, o que não permite investigar a capacidade resolutiva. O foco está na simbologia escrita da equação algébrica. Enquanto que o tipo de reso-lução 2 deixa explícito o raciocínio discreto do aluno.

Lopes Junior (2005) investigou, em sua pesquisa, a compreensão de função de variável discreta de $1^{\circ}$ grau e, revelou que alunos de Ensino Médio não distinguem e não compreendem quando a variável assume valor discreto ou contínuo.

Este fato comprova a importância de praxeologias com abordagens a variáveis discretas e contínuas, bem como discussões em sala de aula tanto da perspectiva da matemática, bem como das aplicações, por exemplo em sistemas digitais (variáveis discretas) e analógicos (variáveis continuas).

\section{Algumas pesquisas na perspectiva da Matemática Discreta na escola básica}

Campbell \& Zazkis (2002), apontam a importância de atividades que promovam o re-investimento de conceitos da Teoria dos Números no ciclo básico, articulados com a resolução de problemas referentes a estes conceitos, de tal forma que não envolvam necessariamente a aplicação direta de algoritmos, mas o desenvolvimento de habilidades como inter-pretar, conjecturar e a busca por estratégias de resolução.

Algumas pesquisas, tais como Freitas (2015), Silva (2014), Campos (2015), Costa (2007), Deus (2017), Silva (2013), Souza (2013) relatam a importância de possibilidades didático-pedagógicas envolvendo a temá- 
tica de Equações Diofantinas Lineares no ensino fundamental e médio. A maioria dessas pesquisas utilizaram como metodologia a resolução de problemas, por exemplo.

As propostas destas pesquisas reafirmam a necessidade em discutir praxeologias que poderiam favorecer a um tipo de raciocinio sobre alguns conceitos matemáticos, por meio do qual os estudantes, futuros professores buscariam estratégias de resolução na perspectiva da Matemática Discreta, tanto nas disciplinas da área pedagógica como Prática de Ensino, quanto nas disciplinas específicas da matemática em um curso de Licenciatura em Matemática.

Nas próximas seções apresentaremos: um contexto histórico das Equações Diofantinas Lineares e do Algoritmo de Euclides, Conexões Matemáticas: Ensino Superior x Escola Básica e, Teorias Matemáticas relativas a estes saberes, de modo a mostrar que existe a possibilidade de discussões sob o ponto de vista da Matemática Discreta entre o ensino superior e a escola básica na formação de professores.

\section{Uma conexão entre o Algoritmo de Euclides e as Equações Diofantinas Lineares - contexto histórico}

Segue um breve relato sobre Diofanto de Alexandria, Euclides e Equações Diofantinas.

\section{Diofanto de Alexandria}

Pouco se sabe sobre a vida de Diofanto de Alexandria, mas muito se atribui a sua importância ao desenvolvimento da álgebra, e segundo Eves (2011, p.207), Diofanto teve grande influência sobre os europeus que posteriormente se dedicaram à teoria dos números. 
De acordo com Bashmakova (1997, p. 1), Diofanto representa um dos enigmas mais dificeis da história da Ciência, pois não se sabe ao certo quando ele viveu. Presume-se que Diofanto nasceu em cerca de 200 d.C., em Alexandria, no Egito, em uma colônia grega e morreu em cerca de 284 d.C., também em Alexandria (FREITAS, 2015, p.18). A suposição para essas datas se dá através de uns versos do matemático Antólios de Alexandria, Bispo de Laodiceia que começou seu episcopado em 270 d.C., e dedicou um livro a seu amigo Diofanto de Alexandria permitindo calcular quantos anos Diofanto viveu:

Deus lhe concedeu ser menino pela sexta parte de sua vida, e somando sua duodécima parte a isso, cobriu-lhe as faces de penugem. Ele lhe acendeu a lâmpada nupcial após uma sétima parte, e cinco anos após seu casamento concedeu-lhe um filho. Ai! Infeliz criança; depois de viver a metade da vida de seu pai, o Destino frio o levou. Depois de se consolar de sua dor durante quatro anos com a ciência dos números, ele terminou sua vida. (FREITAS, 2015, p. 18).

Devido a esse enigma, encontrado no túmulo de Antólio, deduz-se que Diofanto viveu por 84 anos. Ainda, Segundo Freitas (2015), temos a seguinte equação para esse enigma:

$$
\frac{x}{6}+\frac{x}{12}+\frac{x}{7}+5+\frac{x}{2}+4=x
$$

Resolvendo essa equação concluímos que ele viveu por 84 anos.

Assim, como de sua vida, pouco se sabe sobre suas obras, de acordo com Eves (2011, p. 207), Diofanto escreveu três trabalhos: Arithmetica, números poligonais, e prismas. O trabalho de Arithmetica é o mais importante, porém dos 13 livros apenas seis remanesceram, o de números poligonais, sobraram apenas fragmentos e, o trabalho de prismas se perdeu totalmente. 
A importância da Matemática Discreta na formação de professores de Matemática

\section{Euclides}

Sabemos que Euclides foi um dos mais famosos como professor, matemático e escritor da escola de Alexandria. Euclides viveu aproximadamente entre 360 a 295 a.C., porém pouco é conhecido sobre sua vida em Alexandra. É possivel que tenha sido educado em Atenas e teria frequentado a academia de Platão, em pleno vigor da cultura helenística. Sendo assim, convidado para compor o quadro de professores da recémfundada academia, que haveria de tornar Alexandria o centro de todo saber da época. Portanto, logo se tornaria o mais importante autor de matemática da Antiguidade greco-romana e talvez de todos os tempos, com seu monumental Stoichia (Os elementos, 300 a.C.), uma obra composta de treze livros ou capitulos contendo 465 proposições que tratam de tópicos de geometria, teoria dos números e Álgebra (geométrica). Os primeiros quatro livros tratam de geometria plana elementar e estudam propriedades de figuras retilineas e do círculo, sendo abordados problemas cuja solução se faz com régua e compasso. O livro $\mathrm{V}$ tem como abordagem a teoria de proporções e o livro VI faz aplicação dessa teoria ao estudo de geometria. Os livros VII, VIII e IX versam sobre a teoria dos números. O livro X trata dos incomensuráveis e os livros XI, XII e XIII discorrem sobre geometria sólida.

Sobre teoria dos números, em especial as Equações Diofantinas os livros ou capítulos mais abordados são: VII, VIII e IX.

Esses livros são direcionados à teoria dos números, os quais, para os gregos, eram inteiros e positivos. Uma vez que nem todas as grandezas podiam ser representadas por números inteiros, Euclides agregava a cada número um segmento de reta e se referia a ele por AB (FREITAS, 2015, p. 28). Ou seja, as expressões empregadas por ele eram "é medido por" ou "mede". Não utilizando expressões como "é múltiplo de" ou "é fator de". Vinte e duas definições de tipos de números: par e impar, primo e composto, plano e sólido (produto de dois inteiros ou de três inteiros) é encontrado no livro VII. A importância do livro se dá devido as duas primei- 
A importância da Matemática Discreta na formação de professores de Matemática

ras proposições serem o que conhecemos hoje como Algoritmo de Euclides que serve para encontrar o maior divisor comum ou como era representado por ele, a maior medida comum de dois números. No livro IX apresenta a decomposição de um número como produto de fatores primos, a prova de que o conjunto dos números é infinito.

\section{Equações Diofantinas}

A resolução de vários problemas em Matemática conduzem a equações cujas soluções devem ser números inteiros, essas equações do tipo $a x+b y=c$ ou $a x-b y=c$, sendo $a, b$ e $c$ números inteiros não nulos, são denominadas Equações Diofantinas Lineares. Tais equações receberam este nome em homenagem ao matemático grego Diofanto de Alexandria.

De acordo com Sessa (2009), 600 anos ou mais separam Euclides de Diofanto. Nesse periodo, graças às contribuições de diferentes povos a matemática Grega passou por importantes transformações. Para Galvão (2008), deve-se a Diofanto a primeira utilização de símbolos nas equações, pois em suas notações numéricas, usou conforme sua época, as letras do alfabeto grego para simbolizar uma incógnita. Como descrito por Sessa (2009), esse símbolo (uma espécie de S empregados em todos os problemas) designavam quantidades desconhecidas, denominado por Diofanto como "arithmo" (que poderia ser traduzido como número). Diofanto introduziu um símbolo também para a operação de subtração, adição, frações e potência.

Diofanto também formulou regras de transformação de equações que envolveu adição de termos iguais a ambos os lados da equação e redução de termos semelhantes. Mais tarde, essas duas regras se tornaram bem conhecidas sob seus nomes árabes de al-jabr e al-muqabala (Bashmakova, 1997, p. 9) assim podemos perceber a importância de Diofanto para a álgebra e, posteriormente, para a Teoria dos números, dada as equações indeterminadas do tipo $a x+b y=c$, que até hoje são chamadas de Equações Diofantinas. 
Ensino Superior $\times$ Escola Básica: Conexão Matemática Equações Diofantinas Lineares: Conexão com o algoritmo de Euclides para o cálculo do Máximo Divisor Comum (MDC)

A Figura 4 apresenta um esquema desta conexão, a partir do Triângulo Didático, no sentido de observarmos as diversas possibilidades de planejamento de Situações Didáticas sobre os saberes que envolvem o conceito das Equações Diofantinas e, suas soluções. O planejamento de Situações Didáticas poderia ser implementado nos cursos de Licenciatura em Matemática a partir de cada saber que será explicitado nesta seção e suas conexões com a matemática escolar.

Essas discussões sobre Situações Didáticas tendo como fundamento o Triângulo Didático e, as interações professor-aluno-saber, poderiam contribuir com a epistemologia do professor em relação ao saber, de modo a capacitá-lo no processo de transposição didática a fim de estreitar a relação pedagógica entre o professor e o aluno da escola básica e, assim, consequentemente contribuir para uma significativa relação do aluno com o saber.

A partir das conexões entre os tópicos indicados pelas setas de duplo sentido (Figura 4) explicitaremos as Teorias Matemáticas sobre Números Primos, Divisibilidade e Algoritmo Euclidiano e Equações Diofantinas e, suas conexões com os conteúdos matemáticos da escola básica. 
A importância da Matemática Discreta na formação de professores de Matemática

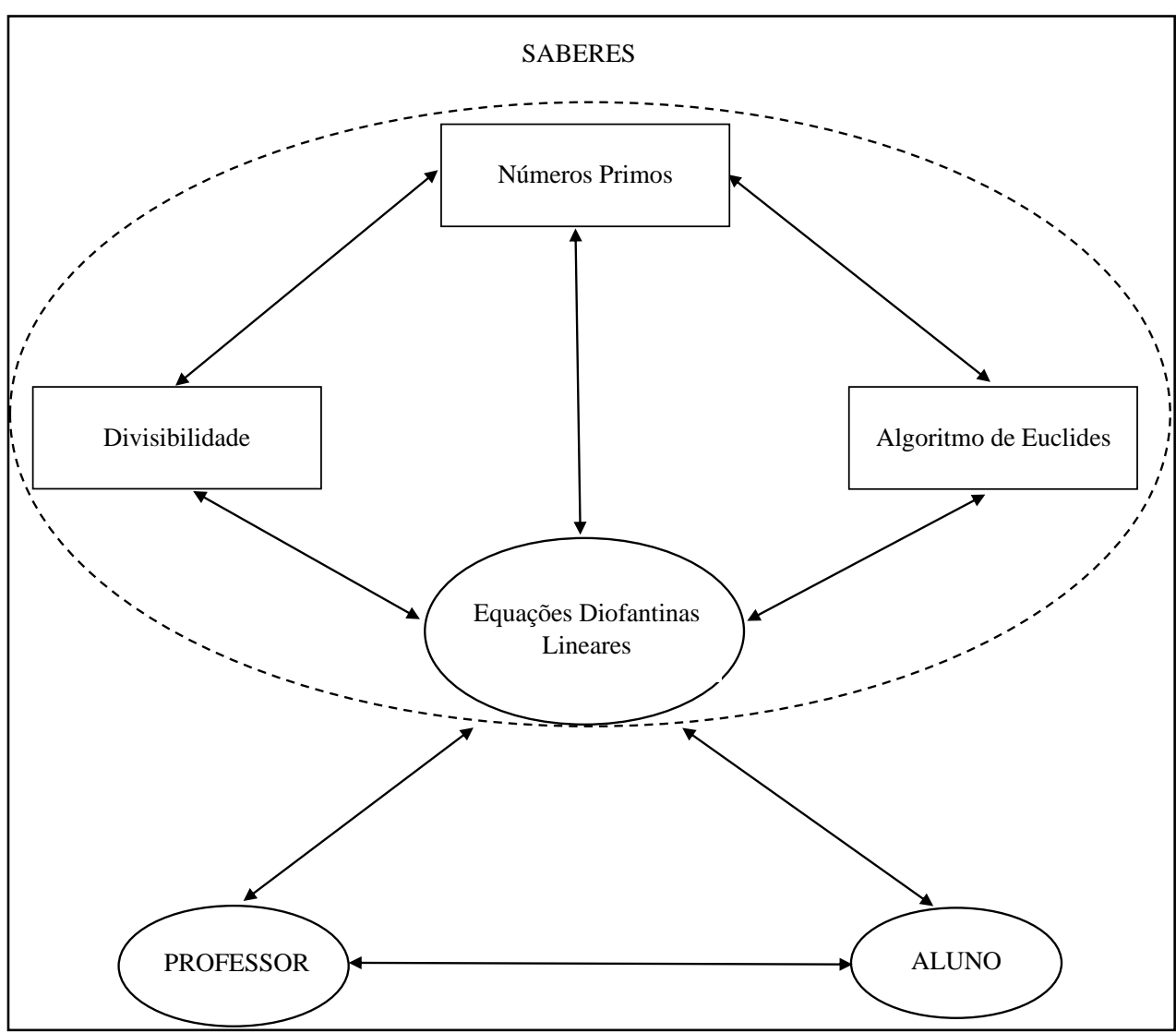

Figura 4. Triângulo Didático

Fonte: Autores

- Números Primos: Classificar Números Naturais em Primos ou Compostos

- Divisibilidade: Divisão Euclidiana, Critérios de Divisibilidade

- Algoritmo euclidiano: (i) Comparação da Eficácia na determinação do Máximo Divisor Comum (MDC) quando comparado aos fatores primos; (ii) Perspectivas / Consolidação: Cálculo de Inversos Multiplicativos.

- Equações Diofantinas: conexão com todos os conteúdos anteriores, progressão aritmética e função afim. 
A importância da Matemática Discreta na formação de professores de Matemática

Essas conexões mostram que existe a possibilidade de discussões e/ou reflexões entre o ensino superior e a escola básica na formação de professores. A seguir, destacamos algumas das Teorias Matemáticas destes saberes, Figura 4 e, alguns exemplos de aplicação direta.

\section{O algoritmo de Euclides para o cálculo do MDC}

Antes de enunciar o algoritmo geral, apresentaremos um método sistemático, ou algoritmo, para encontrar o máximo divisor de dois intei-ros positivos. Esse método é chamado de Algoritmo Euclideano. O nome é devido ao matemático da antiga Grécia, Euclides, que descreve este algoritmo em seus Elementos. ( $\mathrm{O}$ mesmo método para encontrar os maiores divisores comuns também foi descrito no século VI pelo matemático indiano Ariabhata (476 E. C - 550 E. C), que o chamou de "pulverizador").

Segue uma demonstração da utilização deste algoritmo:

Exemplo: Encontrar o maior fator comum de 30 e 72 .

Resolução: Primeiro, usar o algoritmo de divisão para escrever $72=30 \cdot 2+12$, pode-se notar que:

$\operatorname{mdc}(30,72)=\operatorname{mdc}(30,72-2.30)=(30,12)$.

A justificativa para substituir 72 pelo número menor 12 nos cálculos, é porque $m d c(30,72)=m d c(30,12)$. Após essa substituição usar o algoritmo de divisão novamente para escrever $30=2 \cdot 12+6$. Usando o mesmo raciocínio como anteriormente, vemos que $m d c(30,12)=m d c(12,6)$.

Como $12=6.2+0$, pode-se notar que $\operatorname{mdc} \operatorname{mdc}(12,6)=\operatorname{mdc}(6,0)=6$. Consequentemente, pode-se concluir que $\operatorname{mdc}(72,30)=6$, sem encontrar todos os divisores comuns de 30 e 72 . Como geralmente é apresentado na escola básica. 
Essa questão é algo que poderá ser discutido no curso de Licenciatura em Matemática, por exemplo.

Apresentamos agora a forma geral do Algoritmo Euclideano para calcular o máximo divisor comum de dois inteiros positivos.

Teorema 10 Algoritmo de Euclides. Seja $r_{0}=a$ e $r_{1}=b$ inteiros tais que $a \geq b>0$. Se o algoritmo da divisão for sucessivamente aplicado para obter $r_{j}=r_{j+1} q_{j+1}+r_{j+2}$, com $0<r_{j+2}<r_{j+1}$ para $j=0,1,2, \ldots, n-2$ e $\mathrm{r}_{\mathrm{m}+1}=0$, então $\operatorname{mdc}(a, b)=\mathrm{r}_{\mathrm{n}}$, o último resto diferente de zero.

A partir deste teorema, vemos que o máximo divisor comum de $a$ e $b$ é o último resto não nulo na sequência de equações gerada pela aplicação sucessiva do algoritmo de divisão e continuando até o resto ser 0 (onde, em cada etapa, o dividendo e divisor são substituídos por números menores), ou seja, o divisor e o resto.

Para uma prova de que o Algoritmo Euclideano produz o máximo divisor comum, o seguinte lema é utilizado.

Lema 1 Se $c$ e $d$ são inteiros e $c=d q+r$, onde $q$ e $r$ são inteiros, então, $m d c(c, d)=m d c(d, r)$.

O Algoritmo Euclideano é uma maneira extremamente eficaz de encontrar os maiores divisores comuns de dois inteiros.

\section{Expressando o Máximo Divisor Comum como uma Combinação Linear}

O Algoritmo de Euclides também pode ser usado para achar a expressão do $\operatorname{mdc}(a, b)=r_{n}$ como combinação linear de $a$ e $b$, para o que basta eliminar sucessivamente os restos $r_{n-1}, r_{n-2}, \ldots, r_{3}, r_{2}, r_{1}$ entre as $n$ primeiras igualdades anteriores.

Exemplo: Expressar o $\operatorname{mdc}(963,657)=9$ como uma combinação linear de $963 e 657$. 
Essa expressão se obtém eliminando os restos 36,45 e 306 do seguinte modo:

$$
\begin{gathered}
9=45+36(-1) \\
36=306+45 \cdot(-6) \\
45=657+306 \cdot(-2) \\
306=963+657(-1)
\end{gathered}
$$

Substituindo-se sucessivamente esses restos, a parir da segunda equação, na primeira, temos:

$$
\begin{aligned}
9= & 45+36(-1)=45+[306+45 \cdot(-6)](-1)==306(-1)+45(7)= \\
& =306(-1)+[657+306 \cdot(-2)](7)=657(7)+306(-15)= \\
& =657(7)+[963+657(-1)](-15)=963(-15)+657 \cdot(22) .
\end{aligned}
$$

Assim, $9=963(-15)+657 \cdot(22)$.

Reiteramos que essa representação do inteiro $9=\operatorname{mdc}(963,657)$ como combinação linear de963 e 657 não é única. Assim, por exemplo, somando e subtraindo o produto (963)*(657) ao segundo membro da igualdade, obtemos:

$$
\begin{aligned}
9 & =963 \cdot(-15)+657 \cdot(22)+(963) \cdot(657)-(963) \cdot(657)= \\
& =963(-15+657)+657(22-963)= \\
& =96 \cdot(642)+657 \cdot(-941)
\end{aligned}
$$

a qual, é outra representação do inteiro $9=m d c(963,657)$ como combinação linear de 963 e 657.

Isto mostra como mover-se para cima, através das equações que são geradas pelo Algoritmo de Euclides de modo que, em cada etapa, o maior divisor comum de $a$ e $b$ possa ser expresso como uma combinação linear de $a$ e $b$. 
Este método, para expressar o $\operatorname{mdc}(a, b)$ como uma combinação linear de $a$ e $b$, é um pouco inconveniente para o cálculo, pois é necessário elaborar os passos do Algoritmo Euclidiano, salvar todas essas etapas e, em seguida, ir seguindo para trás nas etapas para escrever o $m d c(a, b)$ como uma combinação linear de cada par sucessivo de restos. Existe outro método para encontrar o mdc $(a, b)$ que requer o trabalho através das etapas do método do Algoritmo Euclideano apenas uma vez. A seguir apresentaremos o Teorema 2, que apresenta este método chamado de Algoritmo de Euclides Estendido.

Teorema 2 Sejam $a$ e $b$ números inteiros positivos. Então

$$
\operatorname{mdc}(a, b)=\mathrm{m}_{\mathrm{s}} a+\mathrm{n}_{\mathrm{s}} b
$$

onde $\mathrm{m}_{\mathrm{s}}$ e $\mathrm{n}_{\mathrm{s}}$ são os s-nésimos termos das sequências definidas recursivamente por

$$
\begin{gathered}
\mathrm{m}_{0}=1, \mathrm{n}_{0}=0, \mathrm{~m}_{1}=0, \mathrm{n}_{1}=1, \\
\mathrm{e} \\
m_{j}=m_{j-2}-m_{j-1} q_{j-1}, n j=n_{j-2}-n_{j-1} q_{j-1}
\end{gathered}
$$

para $\mathrm{j}=2,3, \ldots, \mathrm{k}$, onde os $\mathrm{q}_{\mathrm{j}}$ são os quocientes nas divisões no Algoritmo Euclideano quando ele é usado para encontrar o mdc $(a, b)$.

O exemplo a seguir ilustra o uso do Algoritmo de Euclides Estendido. para expressar o $\operatorname{mdc}(a, b)$ como uma combinação linear de $a$ e $b$.

Exemplo: A Tabela 2 apresenta, de forma simplificada, as etapas da implementação do Algoritmo Estendido de Euclides para expressar o mdc $(963,657)$ como uma combinação linear de 963 e 657, sabendo que

$$
\mathrm{mj}=m_{j-2}-m_{j-1} q_{j-1} \text { e } n j=n_{j-2}-n_{j-1} q_{j-1} .
$$


A importância da Matemática Discreta na formação de professores de Matemática

Tabela 2: Etapas do $\operatorname{MDC}(963,657)$

\begin{tabular}{|l|l|c|c|c|l|}
\hline $\mathrm{j}$ & \multicolumn{1}{|c|}{$\mathrm{r}_{\mathrm{j}}$} & $\mathrm{q}_{\mathrm{j}}$ & $\mathrm{m}_{\mathrm{j}}$ & $\mathrm{n}_{\mathrm{j}}$ & \multicolumn{1}{c|}{$\mathrm{r}_{\mathrm{j}}=\mathrm{am}_{\mathrm{j}}+\mathrm{bn}_{\mathrm{j}}$} \\
\hline 0 & $\mathrm{r}_{0}=\mathrm{a}=963$ & & $\mathrm{~m}_{0}=1$ & $\mathrm{n}_{0}=0$ & $963=963(1)+657 .(0)$ \\
\hline 1 & $\mathrm{r}_{1}=\mathrm{b}=657$ & $\mathrm{q}_{1}=1$ & $\mathrm{~m}_{1}=0$ & $\mathrm{n}_{1}=1$ & $657=963(0)+657(1)$ \\
\hline 2 & $\mathrm{r}_{2}=306$ & $\mathrm{q}_{2}=2$ & $\mathrm{~m}_{2}=\mathrm{m}_{0}-\mathrm{m}_{1} \mathrm{q}_{1}=1$ & $\mathrm{n}_{2}=\mathrm{n}_{0}-\mathrm{n}_{1} \mathrm{q}_{1}=-1$ & $\mathrm{r}_{2}=306=963(1)+657(-1)$ \\
\hline 3 & $\mathrm{r}_{3}=45$ & $\mathrm{q}_{3}=6$ & $\mathrm{~m}_{3}=\mathrm{m}_{1}-\mathrm{m}_{2} \mathrm{q}_{2}=-2$ & $\mathrm{n}_{3}=\mathrm{n}_{1}-\mathrm{n}_{2} \mathrm{q}_{2}=3$ & $\mathrm{r}_{3}=45=963(-2)+657(3)$ \\
\hline 4 & $\mathrm{r}_{4}=36$ & $\mathrm{q}_{4}=1$ & $\mathrm{~m}_{4}=\mathrm{m}_{2}-\mathrm{m}_{3} \mathrm{q}_{3}=13$ & $\mathrm{n}_{4}=\mathrm{n}_{2}-\mathrm{n}_{3} \mathrm{q}_{3}=-19$ & $\mathrm{r}_{4}=36=963(13)+657(-19)$ \\
\hline 5 & $\mathrm{r}_{5}=9$ & $\mathrm{q}_{5}=4$ & $\mathrm{~m}_{5}=\mathrm{m}_{3}-\mathrm{m}_{4} \mathrm{q}_{4}=-15$ & $\mathrm{n}_{5}=\mathrm{n}_{3}-\mathrm{n}_{4} \mathrm{q}_{4}=22$ & $\mathrm{r}_{5}=9=963(-15)+657(22)$ \\
\hline 6 & $\mathrm{r}_{6}=0$ & & & & \\
\hline
\end{tabular}

Fonte: Autores

$$
\begin{array}{r}
\text { Como } r_{5}=9=\operatorname{mdc}(963,657) \text { e } r_{5}=m_{5} a+n_{5} b, \text { temos } \\
9=\operatorname{mdc}(963,657)=963(-15)+657 \cdot(22)
\end{array}
$$

A seguir apresentaremos a junção de todas estas teorias aplicadas para obter as soluções de uma Equação Diofantina Linear.

\section{EQUAÇÕES DIOFANTINAS LINEARES}

Quando exigimos que as soluções de uma equação particular sejam do conjunto de inteiros, temos uma Equação Diofantina. Estas equações obtêm seu nome do matemático da antiga Grécia, Diophantus de Alexandria, quem escreveu equações onde as soluções eram restritas aos números racionais. A equação $a x+b y=c$, onde $a, b$ e $c$ são inteiros, é chamada de Equação Diofantina Linear em duas variáveis. 
Observe que o par de inteiros $(x, y)$ é uma solução da Equação Diofantina Linear $a x+b y=c$ se e somente se o par $(x, y)$ é um ponto no plano que se encontra na linha $a x+b y=c$.

A Figura 5 destaca as soluções de $2 x+3 y=5$ em inteiros $x$ e $y$ correspondem aos pontos do plano na reta $2 x+3 y=5$.

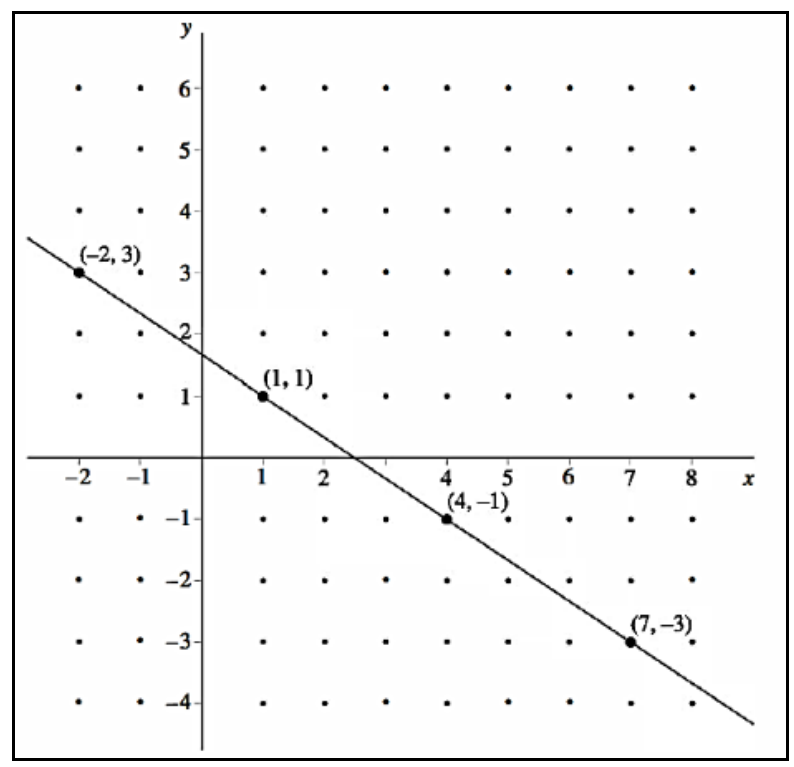

Figura 5. Soluções da equação $2 x+3 y=5$

Fonte: Autores

A primeira pessoa a descrever uma solução geral de Equações Diofantina Linear foi o matemático indiano Brahmagupta (598 - 668), encon-trados nos manuscritos de Aryabhata, um astrônomo e matemático hindu que viveu em cerca de 500 d.C. (BOYER, 1974). A seguir, desenvolveremos as teorias para resolver tais equações.

Lema 2 A Equação Diofantina Linear $a x+b y=c$ tem solução se e somente se $d$ divide $c$, sendo $d=m d c(a, b)$. 
Teorema 2 A Equação Diofantina Linear $a x+b y=c$ tem solução se, e somente se $\mathrm{d} \mid \mathrm{c}$, em que $d=m d c(a, b)$. Se $x_{0}, y_{0}$ é uma solução particular desta equação, então todas as outras soluções são dadas por

$$
x=x_{0}+\frac{b}{d} t, y=y_{0}-\frac{a}{d} t \text {, onde } t \in \mathbb{Z} .
$$

Nota: Uma solução particular da Equação Diofantina Linear se obtém por tentativas ou pelo Algoritmo de Euclides Estendido. E em ambos os casos a solução geral se pode obter usando o Teorema 2, conforme apresentados no exemplo a seguir.

Exemplo: Calcule todas as soluções inteiras e positivas da Equação Diofantina Linear $18 x+5 y=48$.

Resolução: Determinar o mdc $(18,5)$ pelo Algoritmo de Euclides Estendido, resumido na Tabela 3 , lembrando que

$$
x_{j}=x_{j-2}-x_{j-1} q_{j-1} \text {, e } y_{j}=y_{j-2}-y_{j-1} q_{j-1}
$$

Tabela 3: Etapas do mdc(18,5)

\begin{tabular}{|c|c|c|c|c|c|}
\hline$i$ & $r j$ & $q j$ & $x j$ & $y j$ & $r j=a x j+b y j$ \\
\hline 0 & 18 & & 1 & 0 & $18=18(1)+5 \times(0)$ \\
\hline 1 & 5 & 3 & 0 & 1 & $5=18(0)+5(1)$ \\
\hline 2 & 3 & 1 & 1 & -3 & $3=18(1)+5(-3)$ \\
\hline 3 & 2 & 1 & -1 & 4 & $2=18(-1)+5(4)$ \\
\hline 4 & 1 & 2 & 2 & -7 & $1=18(2)+5(-7)$ \\
\hline 5 & 0 & & & & \\
\hline
\end{tabular}

Fonte: Autores (2019) 
Temos, $18 x+5 y=\operatorname{mdc}(18,5)=1$

Multiplicando a equação $1=18(2)+5(-7)$ por 48 , obtemos $48=18(96)+5(-336)$

Logo, o par de inteiros $x_{0}=96, y_{0}=-336$ é uma solução particular da equação proposta, e todas as demais soluções são dadas pelas fórmulas:

$$
x=96+5 t, \quad y=-336-18 t, \text { onde } t \in \mathbb{Z} .
$$

As soluções inteiras e positivas se acham escolhendo t de modo que sejam satisfeitas as desigualdades:

$$
96+5 t>0 \text { e }-336-18 t>0
$$

Isto é:

$$
t>-19,2(-19,-18,-17,-16, \ldots) \text { e } t<-18,6(-19,-20,-21, \ldots)
$$

O que implica $t=-19 \mathrm{e}$, portanto:

$$
x=96+5(-19)=1, y=-336-18(-19)=6
$$

Assim, o par de inteiros $x=1, y=6$ é a única solução inteira e positiva da equação $18 x+5 y=48$, como indicado na Figura 6 .

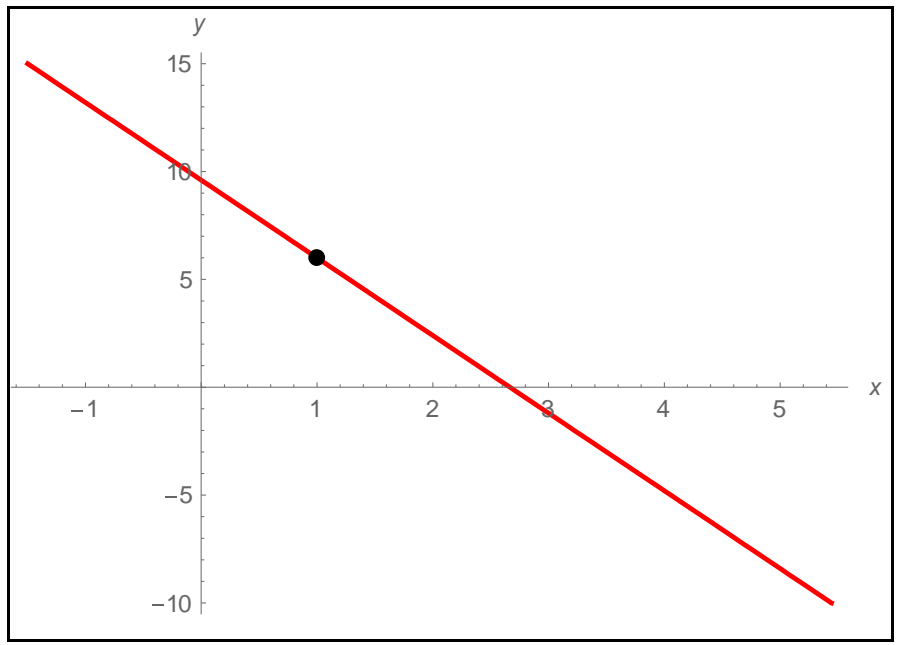

Figura 6. Solução positiva da equação $18 x+5 y=48$

Fonte: Autores

Matemática \& Ciência, v. 3, n. 1, p. 8-37, jun. 2020 - ISSN 2674-9416 
As explanações das teorias matemáticas apresentadas deixam explicitas as conexões apresentadas anteriormente sobre estes saberes em nível do ensino superior e da escola básica, mostrando a importância de reflexões de forma que os alunos se apropriem desses conhecimentos na perspectiva de seu papel como futuro professor.

\section{Considerações Finais}

A partir das pesquisas realizadas e apresentadas neste trabalho procuramos deixar explícita a importância da Matemática Discreta, suas contribuições para o ensino e aprendizagem da matemática pela matemática e, da matemática e suas conexões com outras áreas do conhecimento. Além disso, também apresentamos algumas discussões no sentido de mostrar a possibilidade e importância de um diálogo entre o ensino superior e a escola básica, principalmente no que diz respeito a formação de professores.

Explicitamos aqui apenas algumas aplicações do Algoritmo de Euclides do ponto de vista da matemática pura, mas existem diversas outras aplicações. Como, por exemplo, aplicações do Algoritmo Eucli-diano para gerar os ritmos musicais usados em diferentes culturas em todo o mundo (TOUSSAINT, 2005). Além disso, é um elemento-chave dos algoritmos RSA, um método de criptografia de chave pública usado no comércio eletrônico. Há aplicações na solução de múltiplas congruências (teorema chinês do resto) ou inverso multiplicativo módulo primo. E são aplicações que, se discutidas com os estudantes do curso de Licenciatura em Matemática em sala de aula, os mesmos se apropriarão de conceitos importantes e que contribuirão para a sua atuação como docente na escola básica. Sendo assim, podemos concluir que nossa investigação trouxe subsídios para responder à questão de pesquisa: Quais as contribuições da Matemática Discreta na formação de professores? 
A importância da Matemática Discreta na formação de professores de Matemática

O relato apresentado sobre o contexto histórico das Equações Diofantinas Lineares e do Algoritmo de Euclides; as Conexões Matemáticas: Ensino Superior x Escola Básica e as Teorias Matemáticas relativas a estes saberes, responde a questão de pesquisa: É necessário repensar sobre praxeologias que poderiam favorecer a um tipo de raciocínio por meio do qual os estudantes discorreriam sobre uma maneira de pensar, na perspectiva da Matemática Discreta? Pode-se concluir que as discussões e pesquisas apresentadas mostram a importância do olhar de pesquisadores da área da Educação Matemática e, professores do ensino superior e da escola básica sobre as praxeologias existentes em sua própria instituição de ensino e pesquisa, a fim de repensarem sobre quais tipos de praxeologias poderiam favorecer a um tipo de raciocínio na perspectiva da Matemática Discreta.

\section{Referências}

BASHMAKOVA, Isabella Grigoryevna. Diophantus and Diophantine Equations. New York: Mathematical Association of America, 1997.

BRASIL. Base Nacional Comum Curricular. Brasília: MEC, 2017.

BOYER, C. B. História da Matemática. Tradução de Elza F. Gomide. São Paulo: Edgard Blucher, 1974.

BROLEZZI, A. C. A tensão entre o discreto e o contínuo na história da matemática e no ensino de matemática. $95 \mathrm{f}$. Tese. (Doutorado em Edu-cação) Faculdade de Educação da Universidade de São Paulo, 1996.

CAMPBELL, S.; ZAZKIS, R. Toward number theory as a conceptual field. In: CAMPBELL, S.; ZAZKIS, R. (org.). Learning and teaching Number Theory. London: Ablex Publishing, 2002. Cap 1, p. 1-14.

CAMPOS, A. Equações Diofantinas Lineares: possibilidades didáticas usando a resolução de problemas. 89 f. 2015. Dissertação (Mestrado em Matemática) Programa de Pós-Graduação em Matemática em Rede Nacional, Universidade Federal de Santa, Santa Maria, RS, 2015. 
A importância da Matemática Discreta na formação de professores de Matemática

COMMITTEE ON THE MATHEMATICAL SCIENCES IN 2025. The mathematicalsciences in 2025. Board on mathematical Sciences and their applicationsdivision on Engineering and Physical Sciences - National Research Council. Washington, DC: The National Academies Press, 2013.

CONSElHo NACIONAL DOS PROFESSORES DE MATEMÁTICA. Principios e normas para a Matemática escolar. Lisboa: APM, 2007. (Tradução portuguesa da edição original de 2000).

COSTA, E. S. As Equações Diofantinas Lineares e o professor de matemática do ensino médio. 119 f. 2007. Dissertação (Mestrado em Educação Matemática) - Pontifícia Universidade Católica de São Paulo, São Paulo, 2007.

DeBellis, V. A.; ROSEnstein, J. G. Discrete Mathematics in Primary and Secondary Schools in the United States. ZDM, v. 36 (2), p. 46-55, 2004.

DEUS, N. S. P. Equações Diofantinas Lineares e o GPS: nova conexão curricular. 155 f. Dissertação (Mestrado em Matemática) - Instituto de Matemática, Universidade Federal da Bahia, Salvador, 2017.

DOSSEY, J. Discrete mathematics: the math for our time. In: KENNEY, M. J. \& HIRSCH, C.R. (Eds.). Discrete Mathematics Across the Curriculum, K-12. Reston, VA.: National Council of Teachers of Mathematics, 1991.

EVES, Howard. Introdução a História da Matemática. 5. ed. Campinas, SP: Editora da UNICAMP, 2011.

FREITAS, C. W. A. Equações Diofantinas. 201 f. Dissertação (Mestrado em Matemática) - Programa de Pós-Graduação em Matemática em Rede Nacional, Departamento de Matemática, Universidade Federal do Ceará, Fortaleza, 2015.

GALVÃO, M. E. E. L. História da Matemática: dos números à geometria. Osasco: Edifieo, 2008.

LOPES JUNIOR, D. Função do $\mathbf{1}^{\circ}$ grau: um estudo sobre seus registros de representação semiótica por alunos da $1^{a}$ série do Ensino Médio. 145 f. Dissertação (Mestrado em Educação) - Centro de Ciências Humanas, Universidade Federal de Mato Grosso do Sul, Campo Grande, MS, 2005. 
A importância da Matemática Discreta na formação de professores de Matemática

MENINO, F. S. Resolução de problemas no cenário da matemática discreta. 289 f. Tese (Doutorado) - Instituto de Geociências e Ciências Exatas Universidade Estadual Paulista, São Paulo, 2013.

NATIONAL COUNCIL OF TEACHERS OF MATHEMATICS. Curriculum andevaluation standards for schoolmathematics. Reston, VA: Author, 1989.

OUVRIER-BUFFET, C. Discrete mathematics teaching and learning. In: LERMAN, S. (Ed.). Encyclopedia of Mathematics Education. Dordrecht: Springer, 2014.

SESSA, Carmen. Iniciação ao estudo didático da Álgebra: origens e perspectivas. São Paulo: Edições SM, 2009.

SILVA, A. V. Uso das Equações Diofantinas Lineares no Ensino Fundamental. 74 f. Dissertação (Mestrado em Matemática) - Programa de Pós-Graduação em Matemática. Instituto de Matemática, Universidade Federal de Alagoas, Maceió, 2013.

SILVA, A. S. Um estudo sobre aplicação do algoritmo de Euclides. 68 f. Dissertação (Mestrado em Matemática) - Programa de Pós-Graduação em Matemática, Universidade Federal de Campina Grande, Campina Grande, 2014.

SOUZA, J. R. Matemática discreta: tópicos para o ensino médio. $134 \mathrm{f}$. Dissertação (Mestrado em Matemática) - Programa de Pós-Graduação em Matemática, Universidade Estadual de Londrina, Londrina, 2013.

TOUSSAINT, G. The Euclidean algorithm generates traditional musical rhythms. BANFF CONFRENCE ON THE CONNECTIONS BETWEEN MATHEMATICS AND ART, Music \& Science, 2005, Alberta, Canada, Proceedings... Canadian Mathematical Society, The Banff Centre, PIMS, 2005. p. 47-56 